\title{
The Generalized Matcher Game
}

\author{
${ }^{1}$ Anna Bachstein, ${ }^{1}$ Wayne Goddard, ${ }^{2}$ Connor Lehmacher \\ ${ }^{1}$ School of Mathematical and Statistical Sciences, Clemson University \\ ${ }^{2}$ Department of Mathematics, Vanderbilt University
}

\begin{abstract}
Recently the matcher game was introduced. In this game, two players create a maximal matching by one player repeatedly choosing a vertex and the other player choosing a $K_{2}$ containing that vertex. One player tries to minimize the result and the other to maximize the result. In this paper we propose a generalization of this game where $K_{2}$ is replaced by a general graph $F$. We focus here on the case of $F=P_{3}$. We provide some general results and lower bounds for the game, investigate the graphs where the game ends with all vertices taken, and calculate the value for some specific families of graphs.
\end{abstract}

\section{Introduction}

Fix some graph $F$ with a designated "root" vertex $r$. Given a graph $G$, two players take turns. One player initiates by choosing a vertex $v$, subject to the constraint that $G$ contains at least one (not necessarily induced) copy of $F$ with vertex $v$ corresponding to vertex $r$. The other player responds by choosing one such copy of $F$ within $G$. Vertices can only be used once. This process continues until the remaining vertices of $G$ do not contain a copy of $F$.

One player tries to maximize the number of copies taken. The other player tries to minimize this number. We call these players Maximizer and Minimizer. Thus there are two versions, depending on who initiates and who responds. We define the value of the game as the number of copies taken with optimal play by both players.

This game is a generalization of the matcher game introduced in [1]. That game is where the subgraph $F$ is $K_{2}$. For example, it was shown in [1] that if Maximizer is the responder, then the value of the game is just the matching number of the graph. 
We focus on the graph $P_{3}$. Rooted at the center vertex we call it the 2-star or simply the star; rooted at an end-vertex we call it the stripe. Thus we talk of the star-game and the stripe-game.

We proceed as follows. In Section 2 we provide some examples and elementary results. In Section 3 we determine lower bounds for the game in general graphs and in Section 4 we consider the graphs where the game ends with all vertices taken. In Section 5 we consider some specific families of graphs including grids. Finally in Section 5 we consider the alternative game where there is no "root" vertex.

\section{Examples}

For a graph $G$, we define a $P_{3}$-packing as a collection of vertex-disjoint copies of $P_{3}$ in $G$. Further, we denote by $\mu(G)$ the maximum size of a $P_{3}$-packing of $G$. This parameter generalizes the matching number and is well-studied. For example, Kaneko et al. [2] showed that if $G$ is a 2-connected claw-free graph of order $n$ a multiple of 3 , then $\mu(G)=n / 3$. Earlier, Kirkpatrick and Hell [3] showed that the parameter is NP-complete to compute.

The parameter $\mu(G)$ provides an immediate upper bound on the value of the star- or stripe-game. At the other extreme is the minimum size of a maximal $P_{3}$-packing. It is easy to see that that quantity is at least $\mu(G) / 3$.

As a first example, consider the complete bipartite graph. Note that up to symmetry, the response is forced. So Maximizer as initiator can ensure a maximum $P_{3}$-packing and Minimizer as initiator can ensure a minimum maximal $P_{3}$-packing, regardless of whether it is the star- or stripe-game. Thus:

Lemma 1 Consider the complete bipartite graph $K_{r, s}$ with $r \leq s$ and $s \geq 2$. The value of the game with Maximizer initiating is $\mu=\min (r,\lfloor(s+r) / 3\rfloor)$. The value of the game with Minimizer initiating is $\lceil r / 2\rceil$.

Consider next the game played on a path. If Maximizer initiates, they ensure (almost) all the vertices by choosing an end-vertex for the stripe-game and a 
neighbor of an end-vertex for the star-game. So we consider the version where Maximizer responds.

For the star-game, there is a unique $P_{3}$ for a given central vertex. Thus the game where Minimizer initiates is equivalent to minimum maximal $P_{3}$-packing. The arrangement is to skip two vertices, take a $P_{3}$, skip two vertices, etc. Thus the value of the star-game played on $P_{n}$ is $\lfloor(n+3) / 5\rfloor$ if Minimizer initiates.

Lemma 2 Consider the stripe-game with Maximizer responding. The value of the game played on the path $P_{n}$ is $\lfloor(n+1) / 4\rfloor$.

Proof. Assume the vertices are numbered from 1 up to $n$. Minimizer can ensure at least the claimed value by playing in succession vertex number $2,6,10$, etc. If $n \equiv 3 \bmod 4$, then a final initiation of vertex $n-1$ is invalid, but (both) $n$ and $n-2$ are valid final initiations.

To show that Maximizer as responder can ensure at most the claimed value, it suffices to show by induction that the recurrence relation

$$
f(n)=\min _{1 \leq k \leq n} \max \{f(k-1)+f(n-k-2), f(k-3)+f(n-k)\},
$$

has solution $g(n)=\lfloor(n+1) / 4\rfloor$. For $k^{\prime}=k+4$ it holds that $g\left(k^{\prime}-1\right)+g\left(n-k^{\prime}-\right.$ $2)=g(k-1)+g(n-k-2)$ and that $g\left(k^{\prime}-3\right)+g\left(n-k^{\prime}\right)=g(k-1)+g(n-k)$. So it suffices to check the recurrence for say $1 \leq k \leq 4$. From this it follows that if the recurrence is true for $n$ then it is also true for $n+4$. So it suffices to check the recurrence for four consecutive values of $n$, e.g. $4 \leq n \leq 7$. This can be performed by hand or computer. QED

\section{General Lower Bounds}

\subsection{Lower bounds for Maximizer responding}

Lemma 3 Consider the star-game with Maximizer responding. If $G$ is a graph with $\mu(G)=m$, then the value of the game is at least $\lceil m / 2\rceil$ and this is sharp. 
Proof. For bound: consider a maximum $P_{3}$-packing $\mathcal{P}$ of $G$. Maximizer as responder can ensure that each move overlaps at most two of these copies. If the initiation vertex $v$ is outside $\mathcal{P}$, this is immediate; if $v$ is chosen in some copy of $\mathcal{P}$, then there is an edge to a neighbor of $v$ within the copy, and Maximizer can use that neighbor. Thus the game lasts at least $\lceil m / 2\rceil$ moves.

For optimality: take $m$ copies of $P_{3}$, and pick one end-vertex from each copy and make all the chosen vertices into a path $K$. See Figure 1 where the vertices of $K$ are drawn in white. Minimizer initiates at a vertex in $K$; Maximizer responds with a star using another vertex of $K$ and one degree-2 vertex. QED

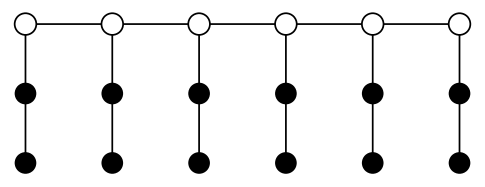

Figure 1: A $P_{3}$-packable graph with smallest value of game for Maximizer responding

Lemma 4 Consider the stripe-game with Maximizer responding. If $G$ is a graph with $\mu(G)=m$, then the value of the game is at least $\lceil m / 2\rceil$ and this is sharp.

Proof. For bound: consider a maximum $P_{3}$-packing $\mathcal{P}$ of $G$. We claim Maximizer as responder can ensure that each move overlaps at most two copies in $\mathcal{P}$. Suppose the initiation is the end-vertex $a$ of a stripe $a b c$ that intersects three copies $Q_{a}, Q_{b}, Q_{c}$ of $P_{3}$ in $\mathcal{P}$. Then vertex $b$ has a neighbor $d$ within $Q_{b}$, so Maximizer can choose the stripe $a b d$ instead. Again the game lasts at least $\lceil m / 2\rceil$ moves.

For optimality: use the same construction as in Figure 1 above. Minimizer plays a vertex adjacent to a leaf; Maximizer is forced to respond with a stripe using two white vertices. QED

\subsection{Lower bounds for Minimizer responding}

Lemma 5 Consider the star-game with Minimizer responding. If $G$ is a graph with $\mu(G)=m$, then the value of the game is at least $\lceil m / 3\rceil$ and this is sharp. 
Proof. For bound: consider a $P_{3}$-packing of $G$ with $m$ copies. No matter what the players do, each move can overlap at most three of these copies. So the game lasts at least $m / 3$ moves. In other words, the lower bound follows from the lower bound on the size of a maximal $P_{3}$-packing noted earlier.

For optimality: consider the "double corona" of the complete graph $K_{m}$, with $m$ a multiple of 3 . That is, take $m$ copies of $P_{3}$ and add an edge between every two center vertices to form clique $K$. See Figure 2, The initiator has to choose a vertex from $K$. Minimizer can respond by taking two more vertices from $K$. The game stops after $\lceil m / 3\rceil$ moves. QED

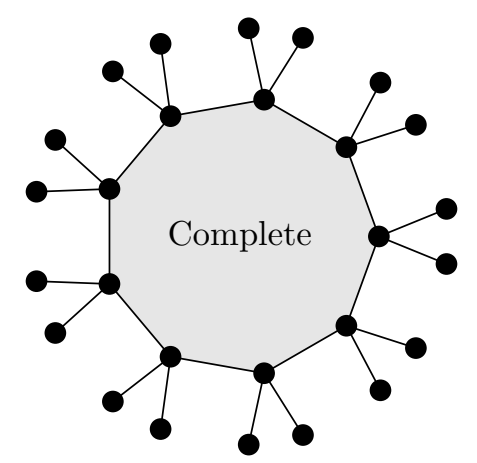

Figure 2: A $P_{3}$-packable graph with smallest value of star-game for Minimizer responding

The above result can be improved for a tree:

Lemma 6 Consider the star-game with Minimizer responding. If $T$ is a tree with $\mu(T)=m$, then the value of the game is at least $\lceil m / 2\rceil$ and this is sharp.

Proof. For bound: consider an optimal $P_{3}$-packing $\mathcal{P}$ of $G$. Consider a vertex $v$ that has at most one non-leaf neighbor. Maximizer initiates at vertex $v$. Then $v$ has at most one edge to a non-leaf, and so Minimizer can overlap at most two stars in $\mathcal{P}$ when responding. Thus the game lasts at least $m / 2$ moves.

Optimality is achieved by the caterpillar formed from $m$ 2-stars by adding edges so that their centers form a path. See Figure 3. QED 


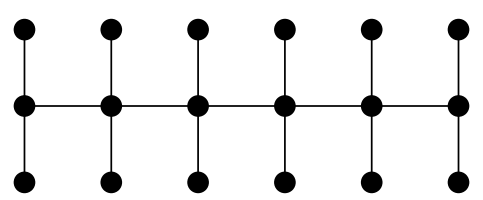

Figure 3: A $P_{3}$-packable tree with smallest value of star-game for Minimizer responding

Consider the stripe-game with Minimizer responding. If $G$ is a graph with $\mu(G)=m$, then by the same trivial argument as before the value of the game is at least $\lceil m / 3\rceil$. It seems unlikely that this bound is achievable in general, but we are unable to prove this. In general, we pose the question:

Question 1 What is the best possible lower bound for the value of the star-game with Minimizer responding as a function of the $P_{3}$-packing number?

As in the case of the star-game, one can prove a better bound if the graph is a tree:

Lemma 7 Consider the stripe-game with Minimizer responding. If $T$ is a tree with $\mu(T)=m$, then the value of the game is at least $\lceil m / 2\rceil$ and this is sharp.

Proof. For bound: consider an optimal $P_{3}$-packing $\mathcal{P}$ of $T$. Maximizer initiates on an end-vertex $v$. Then when Minimizer responds, they have only one option for the neighbor of $v$, and so the stripe taken can overlap at most two copies from $\mathcal{P}$.

Optimality: Consider the caterpillar in Figure 3. When $m$ is even, no matter where Maximizer initiates, Minimizer can respond by using two vertices of the spine and leaving the remaining vertices of the spine to induce paths each with an even number of vertices. The argument when $m$ is odd is similar.

QED

\section{Perfect Graphs}

In the previous section we considered graphs where the value of the game is small. At the other end of the spectrum are those graphs where the number of copies 
taken is the largest it can be. Define a graph as perfect if the result of the game is that all vertices are taken.

If a graph has a $K_{3}$-packing (meaning one can partition the vertex set into triples such that each induces a triangle), then it is immediate that Maximizer as responder can get all the vertices, whether it is the star- or stripe-game. For example, it is easy to build cubic graphs that have a $K_{3}$-packing (just start with a collection of triangles and add a perfect matching).

So we ask for trees: which trees are perfect? In the following we use the fact that a disconnected graph is perfect if and only if each component is perfect.

\subsection{Perfect trees with Maximizer as responder}

Let $\mathcal{D}$ be the family of forests defined as follows. Take some number of disjoint 2 -stars and then add edges between their centers without creating a cycle. (One might call each component the double-corona of a tree.) This includes for example the caterpillar shown in Figure 3 .

Lemma 8 Consider the star-game with Maximizer responding. Then the trees in $\mathcal{D}$ are the perfect trees.

Proof. The graphs in $\mathcal{D}$ are perfect, since Minimizer is forced each time to initiate on a center vertex $v$, and Maximizer can respond by taking $v$ and its two leaves, leaving a member of $\mathcal{D}$.

We argue that these are the only perfect trees. Consider a perfect tree $T$ with an initiation by Minimizer on vertex $a$ and Maximizer's chosen response using vertices $b$ and $c$. Suppose that $b$ is a valid initiation vertex for Minimizer, and Maximizer's response to that would include the new vertex $x$. Let $C_{x}$ be the component of $T-\{a, b, c\}$ containing $x$; this is by assumption $P_{3}$-packable and so has order a multiple of 3 . But if Minimizer initiates on $b$ and Maximizer responds using $x$, then this uses one vertex from $C_{x}$ and so what remains does not have order a multiple of 3 , and so the tree is not perfect. That is, any potential initial vertex $v$ must have two leaf neighbors. Repeat. (Note that the above argument shows that all of $v$ 's other neighbors must have two leaf neighbors, since they too are potential initial vertices.) QED 
Lemma 9 Consider the stripe-game with Maximizer responding. Then $P_{3}$ itself is the only perfect tree.

Proof. If a tree $T$ has more than three vertices, then it contains a non-leaf vertex $v$ that has exactly one non-leaf neighbor. Minimizer initiates on $v$, so that $v$ 's leaf neighbors immediately become isolated. QED

\subsection{Perfect trees with Minimizer as responder}

Let $\mathcal{E}$ be the family of forests defined as follows. Start with some number of $P_{3}$ 's. Repeatedly add a $P_{3}$ and add at most one edge between it and each existing component, except no edge is added incident with the central vertex of the new $P_{3}$. A member of $\mathcal{E}$ is draw in Figure 4.

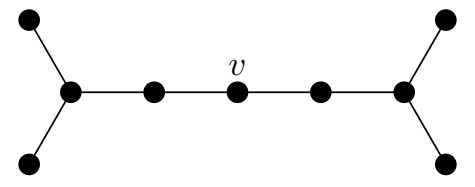

Figure 4: A perfect tree for star-game with Minimizer responding

Lemma 10 Consider the star-game with Minimizer responding. Then the trees in $\mathcal{E}$ are the perfect trees.

Proof. The graphs in $\mathcal{E}$ are perfect, since Maximizer can initiate on the central vertex $v$ of the final added $P_{3}$ and then use recursion. (Minimizer is never given a choice.)

We argue that these are the only perfect trees. Consider a perfect tree $T$ with initiation by Maximizer on a vertex $a$ and assume one possible response is the star bac. Suppose $a$ has degree more than 2 and let $x$ be one of $a$ 's remaining neighbors. Since the component $C_{x}$ of $T-\{a, b, c\}$ containing $x$ is $P_{3}$-packable, it has order a multiple of 3 . If instead Minimizer plays bax, then we have still isolated $C_{x}$ but removed one vertex from it, so it does not have order a multiple of 3 any more, a contradiction. Thus we have shown that Maximizer must initiate on a vertex of degree 2. After removal of the star, apply induction. QED 
Let $\mathcal{F}$ be the family of forests defined as follows. Start with nothing. Repeatedly add a $P_{3}$ with a designated end-vertex $v$, and join $v$ to at most one vertex in each existing component. An example is shown in Figure 5, where the designated end-vertices are numbered in order of creation.

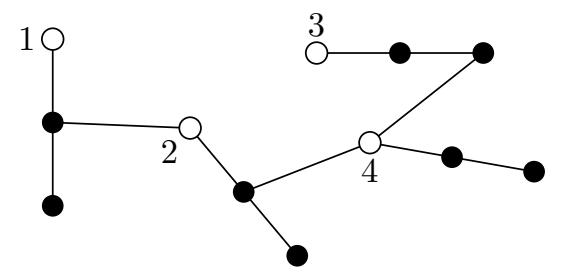

Figure 5: A perfect tree for stripe-game with Minimizer responding

Lemma 11 Consider the stripe-game with Minimizer responding. Then the trees in $\mathcal{F}$ are the perfect trees.

Proof. The graphs in $\mathcal{F}$ are perfect, since Maximizer can initiate on the endvertex of the final added $P_{3}$ that is not $v$, and then use induction/recursion. (Minimizer is never given a choice.)

We argue that these are the only perfect trees. Consider a perfect tree $T$ with initiation by Maximizer on a vertex $a$ and assume one possible response by Minimizer is the stripe $a b c$. Suppose $a$ is not an end-vertex and let $x$ be one of $a$ 's remaining neighbors. Let $C_{x}$ be the component of $T-\{a, b, c\}$ containing $x$. Since it is coverable, $C_{x}$ has order a multiple of 3 ; further $x$ has another neighbor, say $y$. Consider the result if Minimizer responds by taking axy. This removes two of the vertices from $C_{x}$; and so what remains of $C_{x}$, does not have order a multiple of 3 , a contradiction. That is, a must be a leaf.

Suppose now that $b$ has degree more than 2 ; say with another neighbor $z$. Since the component $D_{y}$ of $T-\{a, b, c\}$ containing $z$ is $P_{3}$-coverable, it has order a multiple of 3 . But if the opening move is $a b z$, then we have still isolated $D_{y}$ but removed one vertex from it, so it does not have order a multiple of 3 any more, a contradiction.

Thus we have shown that Maximizer must initiate on a vertex $a$ such that $a$ is end-vertex and its neighbor $b$ has degree 2. After removal of $a b c$, apply induction. QED 


\subsection{Maximal outerplanar graphs}

Recall that a maximal outerplanar graph (MOP) is created by taking a cycle and adding noncrossing chords until their addition is impossible. These graphs are a subset of the 2-trees. We consider which such graphs are perfect.

The triangle $K_{3}$ is always perfect. There are three MOPs of order 6: the fan, snake, and sun, drawn here.
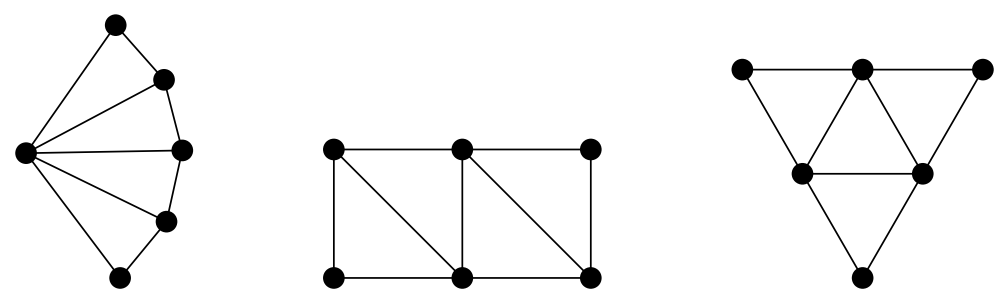

Figure 6: The three MOPs on 6 vertices

Perhaps surprisingly, which is perfect does not depend on the graph but only on the game. Minimizer responding in the stripe-game can ensure the value is 1 ; In the other three games, each graph is perfect. We omit the details. The imperfection generalizes:

Lemma 12 Consider the stripe-game with Minimizer responding. Then the only perfect maximal outerplanar graph is $K_{3}$.

Proof. Assume the graph is perfect and Maximizer initiates at vertex $v$. Suppose that $v$ has degree more than 2 and let $v w$ be a chord incident with $v$. Then removal of $\{v, w\}$ separates the graph $G$ into two components; let $x$ be a neighbor of $w$ on the outer cycle, chosen in the component of order a multiple of 3 if there exists such a component. Then Minimizer plays the stripe $v w x$ and leaves neither component a multiple of 3 , and hence not all vertices are eventually taken. That is, the graph is not perfect.

So vertex $v$ has degree 2. Say its neighbors are $y_{1}$ and $y_{2}$. Then $y_{1}$ and $y_{2}$ have another common neighbor, say $z$. The removal of stripe $v y_{1} z$ creates a component $C$ without $y_{2}$; changing to the removal of stripe $x y_{2} z$ alters the size of $C$ by 1 . So at least one of these removals produces a component not a multiple 
of 3, which gives Minimizer a suitable response to avoid all vertices being taken. That is, the graph is not perfect. QED

It is unclear what the perfect MOPs look like for the other three games. We do note that the first part of the above proof carries over to the star-game on a MOP with Minimizer responding: to have a chance of a perfect outcome, Maximizer must initiate on a vertex of degree 2 .

\section{Some Grid-Like Graphs}

\subsection{Grids with two rows}

Lemma 13 Consider the star-game. The value of the game on a $2 \times m$ grid is $\lceil m / 2\rceil$, regardless of which player has which role.

Proof. Think of the grid as 2 rows and $m$ columns. Consider first the game with Maximizer initiating.

Maximizer as initiator can ensure the desired quantity. Play top row first column, then top row third column, and so on. If $m$ is odd, add one final move of bottom row, last column. Each response is forced: each initiated vertex has exactly two neighbors at the time of being chosen. See Figure 7 .

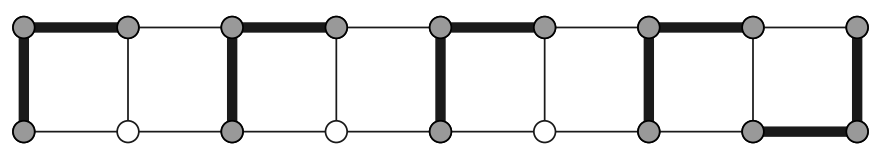

Figure 7: A maximal packing of size 5 in the $2 \times 9$ grid

Minimizer as responder can ensure at most the desired quantity. Assume first that $m$ is even. Minimizer will always use a vertical edge; further, if the star is initiated in column $i$ then: if $i$ is odd, they use the vertex in column $i+1$; and if $i$ is even, they use the vertex in column $i-1$. Equivalently, Minimizer partitions the grid into $2 \times 2$ subgrids, and responds to an initiation in some $2 \times 2$ subgrid by staying within that subgrid. Note that the fourth vertex of the $2 \times 2$ subgrid can never thereafter be chosen by the initiator. 
If $m$ is odd, Minimizer plays the same strategy where possible. Specifically, Minimizer tentatively partitions the grid into $2 \times 2$ subgrids with one "floating" column in the last column. If Maximizer initiates in the last column, then Minimizer uses the vertical edge and one vertex of column $m-1$, as forced. Mentally, Minimizer slides the floating column two to the left and continues the strategy. If initiator plays in the floating column a second time, then again Minimizer uses the vertical edge and the vertex to the left, and slides the floating column two to the left. Eventually the floating column will be surrounded by played $2 \times 2$ subgrids. See Figure 8 for an example. Thus the number of moves is at most one more than the number of $2 \times 2$ grids, which equals $\lceil m / 2\rceil$.

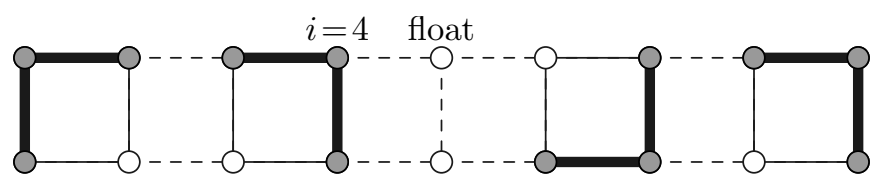

Figure 8: Play in the $2 \times 9$ grid

The analysis of the game with the roles reversed is the same! Minimizer initiating can use the same strategy to end the game in $\lceil m / 2\rceil$ moves. Maximizer responding can use the same strategy to ensure it lasts at least $\lceil\mathrm{m} / 2\rceil$ moves. QED

Lemma 14 Consider the stripe-game. The value of the game on a $2 \times m$ grid is $\lceil m / 2\rceil$ if Maximizer responds and $\lfloor m / 2\rfloor$ if Minimizer responds.

Proof. Minimizer as initiator can ensure at most $\lceil m / 2\rceil$ moves as follows. Play in the top left corner. If Maximizer's stripe is horizontal, then play in the bottom row in the second column and repeat the strategy as if the first four columns are gone. If Maximizer's stripe uses a vertical edge, then repeat as if the first two columns are gone. See FIgure 9. Similarly, Maximizer as initiator can ensure at least $\lfloor m / 2\rfloor$ moves by the same strategy.

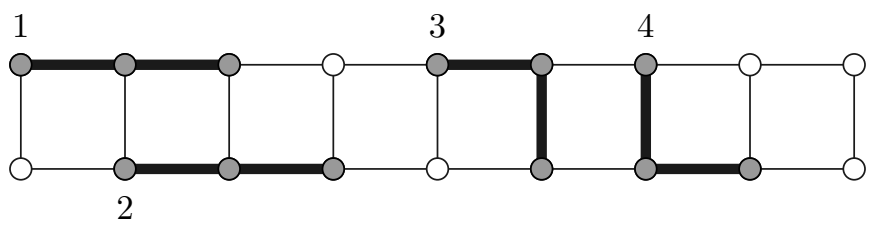

Figure 9: Start of a stripe-game in the $2 \times 9$ grid 
Now, we argue that Minimizer as responder can ensure at most $\lfloor m / 2\rfloor$ moves. Their strategy is as follows. Number the columns 1, 2, up to $m$. Every response, the Minimizer:

uses two vertices from an even-numbered column and one vertex from an odd-numbered column.

We claim Minimizer can always achieve this. If Maximizer initiates with $v$ in an odd-numbered column, and neither even-numbered column next to it is available, then $v$ is not a valid start vertex. If Maximizer initiates with $v$ in an even-numbered column, and Minimizer cannot respond by starting with the vertical edge, then that means the neighboring odd-numbered columns each have a vertex taken (or don't exist), and so the even-numbered columns next over are, by the strategy, taken (or don't exist), and so this is again not a valid move for Maximizer (instead $v$ is the center of a star with 3 leaves).

There are $\lfloor m / 2\rfloor$ even-numbered columns. And so that is an upper bound on the number of moves the Minimizer can be forced to make.

Maximizer as responder can ensure at least $\lceil m / 2\rceil$ moves. This uses a similar idea to above. Specifically:

every response uses two vertices from an odd-numbered column and one vertex from an even-numbered column.

As above, if such a response is not possible, then the initiator chose an invalid vertex. Further, we claim that if there is no valid vertex available for the initiator, then every odd-numbered column is full. The lower bound and the result follows. QED

\subsection{Grids with three or more rows}

Maximizer can do well sometimes on the grid with three rows.

Lemma 15 For the star-game with Minimizer responding, the three-row grid is perfect. 
Proof. Assume we have a grid with three rows and with columns numbered from 1 up to $m$. There are two distinct strategies based on the parity of $m$.

Even case: Maximizer starts in the lower left corner. This gives Minimizer a forced move. Then, Maximizer plays the bottom vertex in the third column, forcing the Minimizer again. This continues with Maximizer playing the bottom vertex in column $2 i+1$ for increasing $i$. Thereafter, Maximizer plays in the top right corner, again forcing the Minimizer, and then moves left across the top row initiating in column $2 i+1$ for decreasing $i$.

Odd case: Here Maximizer starts with the middle vertex in the first column. If Minimizer responds within the column, then we are back in the even case. So without loss of generality assume that Minimizer responds by using the vertex in the second column and the vertex in the bottom left hand corner. Then Maximizer initiates in the top row second column. Minimizer has a forced response. Maximizer continues by playing in column $2 i$ in the top row for increasing $i$, followed by playing along the bottom row in column $m-2 i$ for decreasing $i$. See Figure 10. Each response after the first move is forced. QED

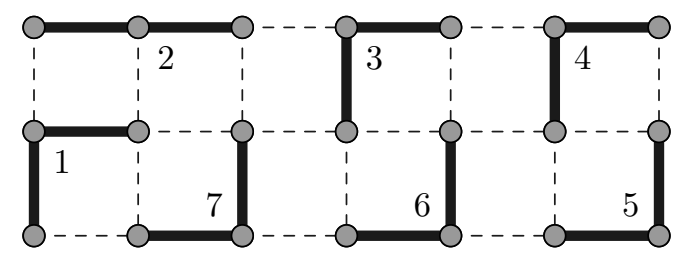

Figure 10: A perfect star-game

It can be shown that for the stripe-game on three-row grid with $m$ columns, with Maximizer as initiator, the value is $m$ if $m \leq 3$, and $m-1$ otherwise. In contrast, the value of either game when Minimizer initiates is asymptotically at most $(1-\varepsilon) m$ for some $\varepsilon>0$. We omit the proofs. (In particular we do not know the optimal $\varepsilon$.)

For general grids, we note that initiator can use the same strategy as in Lemma 13 to achieve $3 / 4$ the vertices being used. But it is unclear whether this is good, bad, or indifferent. 


\subsection{Rooks graphs with two rows}

We consider rooks graph with two rows. That is, the graph $R_{m}$ obtained by taking two cliques of size $m$ and adding a perfect matching between them, which we call cross-edges. For simplicity, we restrict to $m$ a multiple of 3 .

Every maximal $P_{3}$-packing for such graph will leave at most 3 vertices; and if so, the residue will be 2 vertices from one clique and 1 from the other. Thus the value of the game is either $m$ or $m-1$.

If Maximizer is the responder, then they can stay within the row, maintaining each row's size a multiple of 3 , and therefore always get every vertex. That is:

Lemma 16 If Maximizer is responding, then for both games $R_{m}$ is perfect for all $m$ a multiple of 3 .

So we consider the game where Minimizer responds.

Theorem 17 Consider the star-game with Minimizer responding. Then $R_{m}$ is perfect for all $m$ a multiple of 3 .

Proof. Maximizer initiates somewhere. (The graph is vertex-transitive.) There are two cases.

Case 1: Minimizer stays within that row. Then, Maximizer initiates at any vertex that does not have a cross-edge. This forces Minimizer to play within the row, and ensures that the number of vertices in each row remains a multiple of 3 . If every vertex has a cross-edge, then we are back to a rooks graph, and can apply induction; otherwise Maximizer continues with a vertex that does not have a cross-edge.

Case 2: Minimizer uses the cross-edge for the first star. Say Maximizer initiated in the top row, so that two vertices were taken from the top row and one from the bottom row. Then Maximizer plays the vertex in the bottom row that has no cross-edge, to which Minimizer is forced to respond by taking three in the bottom row. Thereafter, Maximizer plays a vertex in the bottom row, and repeats so long as Minimizer stays within that row. 
Eventually, since the number of vertices in the bottom row is not a multiple of 3, Minimizer is forced to use the cross-edge. At that point, both rows have number of vertices left a multiple of 3; thus we are back in Case $1 . \quad$ QED

Theorem 18 Consider the stripe-game, with Minimizer responding. Then $R_{3}$ is perfect but $R_{m}$ for $m \geq 6$ is not.

PROOF. It can easily be checked that $R_{3}$ is perfect: whatever the first move, what is left is connected on 3 vertices and thus can be taken.

Consider $R_{m}$ for $m \geq 6$. Minimizer's strategy will ensure that, until the very end, every vertex in the smaller row still has its cross-edge. Therefore, we can refer to the situation by just the counts of the two rows. We will use $(i, j)$, with $i \geq j$, to denote the situation where one row has $i$ vertices and one row has $j$ vertices. For the base of the induction, we need the case $(4,2)$. For this, one can readily check that wherever Maximizer initiates, Minimizer can respond and disconnect the graph, thereby ending the game.

For $R_{m}$ the play starts at the case $(m, m)$. For the first move, Minimizer uses a cross-edge; so the case becomes $(m-1, m-2)$. We claim that Minimizer can ensure the case $(m-2, m-4)$ next. For, if Maximizer chooses a vertex in the larger side, then Minimizer stays in the larger side, using up the vertex that has no cross-edge; and if Maximizer chooses a vertex in smaller side, then Minimizer takes two vertices there and a cross-edge to the other row.

We claim that Minimizer can ensure the case $(m-4, m-5)$ next. For, if Maximizer initiates on the larger side, then Minimizer takes three vertices there; and if Maximizer initiates on the smaller side, then Minimizer immediately uses the cross-edge and then takes one of the (two) vertices without a cross-edge. By repeated application of the strategy, Minimizer can alternate between cases of the form $(x, x-2)$ and $(y, y-1)$ until they reach the case $(4,2)$, which we saw is not perfect. QED 


\section{The Unrooted $P_{3}$}

There is also a version of the game where the packing subgraph has no root. We define the unrooted- $P_{3}$-game to be the game where responder need only choose a copy of $P_{3}$ containing the designated vertex. We show that in some cases the value of the game is the same as in the rooted version, but in other cases it is different. In particular, for Maximizer responding, they get all that is possible.

\subsection{Maximizer responding}

Theorem 19 Consider the unrooted-P $P_{3}$-game with Maximizer responding played on graph $G$. Then the value of the game is $\mu(G)$.

Proof. Consider a maximum $P_{3}$-packing $\mathcal{P}$ of $G$. If Minimizer chooses a vertex $u$ in $\mathcal{P}$, then Maximizer responds with the associated copy in $\mathcal{P}$ and repeats. If instead Minimizer chooses a vertex $v$ outside $\mathcal{P}$, then by requirement, the vertex $v$ is in a copy $Q$ of $P_{3}$. The only way a problem could arise is if $Q$ intersects two copies in $\mathcal{P}$. But that implies that vertex $v$ has an edge to some copy in $\mathcal{P}$; and so Maximizer can use that edge and one edge from that copy to build a $P_{3}$, thereby affecting only one copy in $\mathcal{P}$. Repeat. QED

The above theorem generalizes Theorem 1 on the matcher game from [1]. Note that this pattern does not continue much further. In particular, the star

$K_{1,3}$ does not have a similar result. For example, take three copies of $K_{1,3}$ and add a new vertex $v$ adjacent to one end-vertex from each copy. The resulting tree has three disjoint $K_{1,3}$ 's, but Minimizer ends the game in one move by initiating on $v$.

\subsection{Minimizer responding}

When one changes to the unrooted game, this gives both player more options. We saw above that when Maximizer is responder, the added options to Minimizer do not help them. A similar result holds if we go from the stripe-game to the unrooted game with Minimizer as responder: 
Theorem 20 Consider Minimizer responding. The value of the unrooted- $P_{3}$ game is at most the value of the stripe-game.

Proof. Consider changing from the stripe-game to the unrooted game. Minimizer plays just as if it were the stripe-game. We argue that the new options do not help Maximizer. For, the only additional option they have is to play a vertex $v$ that is in a $P_{3}$ but is not the end of one. That is, the only additional initiation option they get is to play the center vertex of a star component; but that is equivalent to initiating at a leaf of the component, which they could do already. QED

In contrast, the star-game is incomparable with the unrooted- $P_{3}$-game. Consider, for example, the double corona of a complete graph (see Figure 2). Maximizer now can initiate on a leaf, and thereby ensure that approximately half the vertices are used. On the other hand, consider the tree shown in Figure 4. As we saw, Maximizer can obtain every vertex in the star-game by initiating on the central vertex $v$. In the unrooted $P_{3}$-game, however, Minimizer can respond differently and destroy the perfection.

\section{Questions}

We conclude with some questions for future study. Obviously, a natural direction is to replace $P_{3}$ by another required subgraph. For the games with $P_{3}$, it would be interesting to determine the value of the game on a general grid and a general rooks graph. Another question, is whether there is a $\varepsilon>0$ such that all graphs of order $n$ with minimum degree at least $(1-\varepsilon) n$ are perfect.

\section{References}

[1] W. Goddard and M.A. Henning. The matcher game played in graphs. Discrete Appl. Math. 237 (2018), 82-88.

[2] A. Kaneko, A. Kelmans, and T. Nishimura. On packing 3-vertex paths in a graph. J. Graph Theory 36 (2001), no. 4, 175-197. 
[3] D.G. Kirkpatrick and P. Hell. On the complexity of general graph factor problems. SIAM J. Comput. 12 (1983), no. 3, 601-609. 